EDITORIAL

Rev Obstet Ginecol Venez. 2020; 80 (4): 263-267

https://doi.org/10.51288/00800403

\title{
COVID-19, vacunas y embarazo
}

\author{
Dra. Mireya González Blanco
}

Directora/Editora

Es todo un desafío escribir sobre la covid-19 y su evolución, sobre el agente causal, SARS-CoV-2, que todavía es mencionado en la literatura médica como el nuevo coronavirus, y sobre las importantes medidas de prevención, pero lo es más, cuando la población involucrada en la gestante. En pocos meses, se produjeron y siguen produciéndose miles de artículos que abarcan todas las ramas científicas, que son imposibles de seguir y conocer adecuadamente (1).

Los países y las regiones se encuentran actualmente en diferentes etapas de la epidemia. Durante estos casi 9 meses, el personal sanitario, los investigadores, las organizaciones científicas y gubernamentales y la población general han aprendido mucho sobre el tema. Los procesos de recabar y difundir información son muy dinámicos, de forma que lo que se afirmaba como realidad hace 6 meses, se ha descartado en la actualidad. Sin embargo, una información se ha mantenido como premisa fundamental en la lucha contra la enfermedad y se refiere a la importancia de la prevención. El uso de la mascarilla, el adecuado lavado de manos y el distanciamiento físico, así como evitar los lugares concurridos y los espacios cerrados, son medidas extremadamente eficientes para reducir el contagio. Sin embargo, pareciera que son insostenibles a largo plazo. La identificación, mediante la aplicación de pruebas moleculares masivas, de enfermos, portadores asintomáticos y presintomáticos que son contactos de casos positivos, también es claramente una estrategia útil para reducir la tasa de nuevas infecciones al tiempo que permite la relajación de las medidas de contención.

La intención en esta ocasión es abordar el tema de la prevención en una población particular: las gestantes. Esto resulta de gran importancia porque involucra no solo a la madre, sino también a su feto $y$, después, al recién nacido. En brotes anteriores de otras infecciones por coronavirus, como el síndrome respiratorio agudo grave y el síndrome respiratorio de Oriente Medio, se notificaron complicaciones graves en mujeres embarazadas $(2,3)$, pero, a pesar de que hay más de 54 millones de casos de covid-19 en todo el mundo, la comprensión del efecto del SARSCoV-2 en gestantes, fetos y bebés es incompleta o limitada (2). Se ha informado que una alta proporción de mujeres con infección covid-19 confirmada presenta parto prematuro y parto por cesárea; las tasas estimadas de admisión a la unidad de cuidados intensivos entre mujeres embarazadas son superiores a las de las mujeres no embarazadas y alrededor del $1 \%$ - $9 \%$ de los bebés nacidos de estas mujeres resultan positivos para SARS-CoV-2 (2 - 4). A la fecha, muchas preguntas importantes siguen sin respuesta, incluyendo el alcance de la infección asintomática o leve y el efecto de la covid-19 en el aborto espontáneo, la restricción del crecimiento fetal intrauterino, las anomalías congénitas, el crecimiento a largo plazo y los resultados del neurodesarrollo (2). Aunque algunos autores han señalado que las mujeres embarazadas no se consideran con mayor riesgo de complicaciones en comparación con las mujeres no embarazadas, parecen tener, al menos, un riesgo similar $(2,3)$, sin embargo, otros describen que existe un creciente número de pruebas que sugieren que las mujeres corren un mayor riesgo de morbilidad y mortalidad por covid-19 durante el embarazo, incluido un mayor riesgo de insuficiencia respiratoria con la necesidad de admisión a cuidados intensivos y ventilación mecánica, en comparación 
con las mujeres fuera del embarazo; además, la enfermedad se ha asociado con una mayor tasa de mortinato (5). A pesar de las diferentes conclusiones existentes, la evidencia parece inclinarse hacia que la covid-19 no es particularmente agresiva en mujeres embarazadas, neonatos o niños, sobre la base de datos limitados disponibles. Debido a la naturaleza dinámica y la rápida evolución de la comprensión científica de esta condición, es probable que estas premisas cambien a medida que crece el conocimiento (6).

Dada su susceptibilidad ante la infección, las mujeres embarazadas deben ser consideradas candidatas a medidas preventivas, de las cuales la vacunación es el estándar de oro (2) que genera grandes expectativas porque una vacuna eficaz representa la posible solución definitiva a la enfermedad. Actualmente hay muchas candidatas a vacuna, incluidas algunas en ensayos de fase tres. Hasta que exista una vacuna, es probable que el SARS-CoV-2 siga causando brotes que requieran nuevas rondas de cierre social, protección a grupos susceptibles y uso obligatorio de mascarillas en espacios públicos y eventos. Es de esperar que esta tragedia mejore la cooperación internacional, potencie a las organizaciones multinacionales y resalte al mundo la importancia de la ciencia (1).

La complejidad del sistema inmunitario materno surge de su capacidad para prevenir una respuesta inmune materna contra un feto semialogénico, al tiempo que protege a la madre contra patógenos dañinos. Sin embargo, estas adaptaciones inmunológicas también pueden hacer que las mujeres embarazadas sean vulnerables a desarrollar complicaciones adversas de infecciones virales respiratorias y que puedan no responder a las vacunas con la mayor eficacia. Mientras que la mayoría de las vacunas virales son capaces de inducir una respuesta seroprotectora de anticuerpos, en algunas personas de alto riesgo esto puede no correlacionarse con la protección clínica. Algunos estudios han demostrado que factores como la edad, el sexo y las enfermedades crónicas pueden reducir la respuesta ante la vacuna y cabría evaluar cómo el embarazo puede afectar su eficacia e inmunogenicidad (7).

En las últimas semanas y meses, varias candidatas a vacunas covid-19 han entrado en fase clínica. $\mathrm{Si}$ bien es esencial el desarrollo de las vacunas covid-19 a tiempo para usarlas para esta pandemia actual, también existen posibles problemas de seguridad que podrían ralentizar el desarrollo clínico y las pruebas (8). La vacuna debe proporcionar beneficios altamente favorables, con elevada eficacia, solo efectos adversos leves o transitorios, sin efectos adversos graves. La vacuna debe ser adecuada para todas las edades, mujeres embarazadas y lactantes; debe proporcionar un inicio rápido de la protección con una sola dosis y conferir seguridad durante al menos un año de administración (9). Los trabajadores de la salud, las personas mayores de 65 años y las personas con enfermedades cardiovasculares, enfermedades respiratorias crónicas, cáncer, diabetes u obesidad serán priorizados para la vacunación inicial. Llama la atención que las mujeres embarazadas no parecen constituir un grupo de alta prioridad.

Las estrategias de vacunación se pueden utilizar eficazmente para reducir la gravedad de la enfermedad, controlar la transmisión mediante la diseminación viral y prevenir futuras infecciones (10). Se han realizado muchos esfuerzos en el desarrollo de las vacunas contra covid-19, la mayoría de las candidatas a vacunas en desarrollo utilizan la proteína $\mathrm{S}$ de SARSCoV-2 (9). El proceso de desarrollo de vacunas es bastante laborioso con varias etapas, incluyendo la etapa preclínica y el desarrollo clínico que es un proceso trifásico. Sin embargo, si ya se dispone de datos suficientes, se ha recomendado omitir algunas etapas, para acelerar el logro de una vacuna con una rápida revisión regulatoria, aprobación, fabricación y control de calidad, por lo tanto, este nuevo coronavirus ha obligado a la comunidad científica a utilizar enfoques no convencionales para acelerar el proceso de desarrollo de vacunas (9). 
Existen más de 150 candidatas a vacunas, de las cuales, la mayoría se encuentra en fase preclínica o exploratoria, pero unas cuantas se encuentran en fase tres de investigación clínica; incluso, recientemente, dos grandes farmacéuticas anunciaron que las vacunas que desarrollan contra la covid-19 tienen un $90 \%$ y $94 \%$ de efectividad para prevenir la enfermedad en personas sin evidencia de infección previa (11, 12). Estas vacunas experimentales comprenden una serie de tecnologías innovadoras y tradicionales, que van desde ácidos nucleicos envasados y vectores de virus hasta proteínas recombinantes combinadas con adyuvantes, algunas se basan en virus atenuados, subunidades proteicas, partículas similares a virus (VLP), vectores virales (replicantes y no replicantes), ADN, ARN, nanopartículas, etc. y cada una presenta ventajas y características únicas $(8-10)$.

Una vez que se tenga la vacuna será necesario preparar cuidadosamente una política eficaz para desarrollar un marco de acceso mundial para una distribución equitativa en el mundo en desarrollo, protegiendo al mismo tiempo la propiedad intelectual y los derechos de mercado. La distribución de las vacunas debe hacerse en la medida de lo posible sobre la base de la benevolencia humanitaria, a través de los organismos internacionales competentes (10).

Un componente crítico en el manejo de cualquier amenaza de enfermedad transmisible es la atención de las poblaciones vulnerables. Como se señaló previamente, las mujeres embarazadas se ven afectadas desproporcionadamente por enfermedades respiratorias, que se asocian con un aumento de la morbilidad infecciosa y altas tasas de mortalidad materna. Por ello, representan un grupo especialmente vulnerable en cualquier brote de enfermedades infecciosas debido a su fisiología alterada, susceptibilidad a las infecciones y sus funciones mecánicas e inmunológicas comprometidas. La necesidad de salvaguardar al feto se suma al desafío de gestionar su salud (13).
Se han identificado una serie de estrategias específicas para promover la inclusión éticamente responsable, socialmente justa y respetuosa de los intereses de las mujeres embarazadas en el desarrollo y evaluación de vacunas contra patógenos emergentes (2). La necesidad de una vacuna contra covid-19 es indiscutible, es una prioridad sanitaria mundial $(2,9)$, sin embargo, una consideración particular es que para muchas de las candidatas y plataformas vacunales que se están considerando activamente, como ácidos nucleicos, vectores virales y nuevos adyuvantes, no se han realizado ensayos en mujeres embarazadas $(2$, 6). Las gestantes, por tanto, se verían perjudicadas si no pueden acceder a la quimioprofilaxis basada en la evidencia de los ensayos de vacunas. Por consiguiente, el compromiso mundial para el acceso equitativo a las vacunas covid-19 debería incluir a las mujeres embarazadas, desarrollando protocolos que incluyan disposiciones para el seguimiento de la seguridad materna y fetal y para la documentación de las complicaciones iatrogénicas, incluido el seguimiento de la descendencia después del parto (5). Con respecto a este planteamiento, habría que sopesar los riesgos y beneficios. Por un lado, no incluir a la gestante en los ensayos clínicos de fase tres evitaría los riesgos de efectos adversos sobre la mujer y la evolución de su embarazo, el feto y el recién nacido, por el otro, la gestante no incluida en los ensayos de vacunación no se beneficiaría de la evaluación de la eficacia y la seguridad en esa población específica.

Hay pruebas emergentes de que la eficacia de la vacuna podría verse influenciada negativamente por el embarazo. Los nuevos métodos para medir la eficacia de la vacuna en grupos de alto riesgo, incluido el embarazo, pueden ayudar a determinar si la respuesta realmente se reduce (7). Dado que las respuestas inmunitarias a la vacunación en mujeres embarazadas no pueden asumirse a partir de las de aquellas que no lo están y que la evaluación de la seguridad de la vacunación en el embarazo es única, las gestantes deberían incluirse en ensayos de vacunas adecuadamente diseñados (2). El embarazo proporciona un desafío complejo 
para el sistema inmunitario materno, ya que necesita proteger a la madre contra las infecciones, al tiempo que crea tolerancia inmune a los antígenos paternos y al trofoblasto (7). La inclusión de estas mujeres y sus recién nacidos garantizará que puedan beneficiarse de las candidatas a vacunas que resulten exitosas y que, en última instancia, estarán protegidas contra covid-19 (2). De no incluirlas, probablemente se verían limitadas al uso de las medidas de protección general durante toda la gestación, obligándolas a posponer la vacunación para después del parto o, incluso, después de la lactancia.

Aunque las vacunas son una de las defensas más poderosas de la salud pública contra las enfermedades infecciosas, históricamente las gestantes han sido excluidas de la investigación y el desarrollo de las mismas. Existe un consenso de expertos en torno a la necesidad crítica de incluirlas en los esfuerzos de desarrollo de vacunas; las directrices recientes ofrecen un camino éticamente sólido hacia adelante para el programa de investigación de vacunas (14). Ahora bien, para permitir su inclusión en los ensayos, es necesario considerar la carga de la enfermedad a corto y largo plazo para esa población específica, el deseo de las gestantes de ser vacunadas contra covid-19 y de participar en los ensayos y, finalmente, la información sobre cuáles de las vacunas candidatas son adecuadas para mujeres embarazadas y deben ser el centro de los primeros ensayos clínicos $(2,14)$.

La pandemia covid-19 destaca la vulnerabilidad de las mujeres embarazadas enfermas si se excluyen sistemáticamente de los ensayos clínicos, y potencialmente limita su acceso a terapias a través de un uso compasivo. Los registros clínicos podrían recopilar datos sobre exposiciones, pero no permitirán a las mujeres embarazadas acceder a la atención basada en la evidencia informada por los ensayos clínicos. Además, la vacunación durante el embarazo protege a la madre, al feto y al recién nacido. Esta triplicación de beneficios significa que el rápido desarrollo de las vacunas debe permitir a las mujeres embarazadas una inclusión segura y oportuna en los ensayos $(7,15)$.
Mientras tanto, es necesario insistir en la aplicación de las medidas preventivas generales que dependen de la población misma: el uso de la mascarilla, el adecuado lavado de manos, el distanciamiento físico y evitar espacios concurridos y cerrados. Cumpliéndolas, llegaremos con bien al momento de la vacunación masiva, que debería representar el fin de la pandemia.

\section{REFERENCIAS}

1. Romano CM, Chebabo A, Levi JE. Past, present, and future of covid-19: a review. Braz J Med Biol Res. 2020; 53(9):e10475. doi: 10.1590/1414-431×202010475.

2. Heath PT, Le Doare K, Khalil A. Inclusion of pregnant women in covid-19 vaccine development. Lancet Infect Dis. 2020; 20(9):1007-1008.

3. Khalil A, Kalafat E, Benlioglu C, et al. SARS-CoV-2 infection in pregnancy: a systematic review and metaanalysis of clinical features and pregnancy outcomes. EClinicalMedicine 2020; published online July 3. https://doi.org/10.1016/j.eclinm.2020.100446.

4. Sutton D, Fuchs K, D'Alton M, Goffman D. Universal screening for SARS-CoV-2 in women admitted for delivery. N Engl J Med 2020; 382: 2163-64

5. Dashraath P, Nielsen-Saines K, Madhi SA, Baud D. covid-19 vaccines and neglected pregnancy. Lancet. 2020; 396(10252):e22. doi: 10.1016/S01406736(20)31822-5.

6. Elgin TG, Fricke EM, Hernandez Reyes ME, Tsimis ME, Leslein NS, Thomas BA, Sato TS, McNamara PJ. The changing landscape of SARS-CoV-2: Implications for the maternal-infant dyad. J Neonatal Perinatal Med. 2020; 13(3):293-305. doi: 10.3233/NPM-200460.

7. Saeed Z, Greer O, Shah NM. Is the Host Viral Response and the Immunogenicity of Vaccines Altered in Pregnancy? Antibodies (Basel). 2020; 9(3):38. doi: 10.3390/antib9030038.

8. Hotez PJ, Corry DB, Bottazzi ME. covid-19 vaccine design: the Janus face of immune enhancement. Nat Rev Immunol. 2020; 20(6):347-348. doi: 10.1038/ s41577-020-0323-4.

9. Kaur SP, Gupta V. covid-19 Vaccine: A comprehensive status report. Virus Res. 2020; 288:198114. doi: 10.1016/j.virusres.2020.198114.

10. Mukherjee R. Global efforts on vaccines for covid-19: 
Since, sooner or later, we all will catch the coronavirus. J Biosci. 2020; 45(1):68. doi: 10.1007/s12038-02000040-7.

11. Meredith S. Pfizer, BioNTech say Covid vaccine is more than $90 \%$ effective-great day for science and humanity. CNBC. 9 de noviembre 2020 [consultado 10 de noviembre de 2020]; Health and Science. Disponible en: https://www.cnbc.com/2020/11/09/covid-vaccinepfizer-drug-is-more-than-90percent-effective-inpreventing-infection.html

12. Lovelace B, Higgins-Dunn N. Moderna says preliminary trial data shows its coronavirus vaccine is more than 94 effective, shares soar. CNBC. 16 de noviembre 2020 [consultado 16 de noviembre de 2020]; Health and Science. Disponible en: https://www. cnbc.com/2020/11/16/moderna-says-its-coronavirusvaccine-is-more-than-94percent-effective.html
13. Dashraath P, Wong JLJ, Lim MXK, Lim LM, Li S, Biswas A, Choolani M, Mattar C, Su LL. Coronavirus disease 2019 (covid-19) pandemic and pregnancy. Am J Obstet Gynecol. 2020; 222(6):521-531. doi: 10.1016/j. ajog.2020.03.021.

14. Jaffe E, Lyerly AD, Goldfarb IT. Pregnant women's perceptions of risks and benefits when considering participation in vaccine trials. Vaccine. 2020; 38(44):6922-6929. doi: 10.1016/j.vaccine.2020.08.059.

15. Whitehead CL, Walker SP. Consider pregnancy in covid-19 therapeutic drug and vaccine trials. Lancet. 2020; 395(10237):e92. doi:10.1016/S01406736(20)31029-1. 